\title{
AMOEBAS OF HALF-DIMENSIONAL VARIETIES
}

\author{
GRIGORY MIKHALKIN
}

\begin{abstract}
An $n$-dimensional algebraic variety in $\left(\mathbb{C}^{\times}\right)^{2 n}$ covers its amoeba as well as its coamoeba generically finite-to-one. We provide an upper bound for the volume of these amoebas as well as for the number of points in the inverse images under the amoeba and coamoeba maps.
\end{abstract}

\section{Introduction}

1.1. Definitions. Consider an $n$-dimensional algebraic variety $V \subset$ $\left(\mathbb{C}^{\times}\right)^{2 n}$.

Definition 1.1 (Gelfand-Kapranov-Zelevinsky [4]). The amoeba $\mathcal{A}$ of $V$ is the image

$$
\mathcal{A}=\log (V) \subset \mathbb{R}^{2 n}
$$

of $V$ under the coordinatewise logarithm map $\log :\left(\mathbb{C}^{\times}\right)^{2 n} \rightarrow \mathbb{R}^{2 n}$,

$$
\log \left(z_{1}, \ldots, z_{2 n}\right)=\left(\log \left|z_{1}\right|, \ldots, \log \left|z_{2 n}\right|\right) .
$$

The restriction $\left.\log \right|_{V}$ is called the amoeba map for $V$.

Definition 1.2 (cf. Passare [15]). The coamoeba (or alga, cf. [3]) $\mathcal{B}$ of $V$ is the image

$$
\mathcal{B}=\operatorname{Arg}(V) \subset\left(S^{1}\right)^{2 n}
$$

of $V$ under the coordinatewise argument map Arg : $\left(\mathbb{C}^{\times}\right)^{2 n} \rightarrow(\mathbb{R} / 2 \pi \mathbb{Z})^{2 n} \approx$ $\left(S^{1}\right)^{2 n}$

$$
\operatorname{Arg}\left(z_{1}, \ldots, z_{2 n}\right)=\left(\arg \left(z_{1}\right), \ldots, \arg \left(z_{2 n}\right)\right) .
$$

The restriction $\left.\operatorname{Arg}\right|_{V}$ is called the coamoeba map for $V$.

For coamoebas it is often more convenient to use argument taken $\bmod \pi$ instead of $\bmod 2 \pi$, (cf. [12]). Namely, we denote $T_{\pi}=\mathbb{R} / \pi \mathbb{Z}$ and for $z \in \mathbb{C}^{\times}$we define

$$
\arg _{\pi}(z)=(\arg (z) \bmod \pi) \in \mathbb{T}_{\pi} .
$$

Research is supported in part by the grant TROPGEO of the European Research Council, by the grants 140666 and 141329 of the Swiss National Science Foundation, and by the NCCR SwissMAP of the Swiss National Science Foundation. 
In other words, $\arg _{\pi}$ is the composition of arg and the double covering $\mathbb{R} / 2 \pi \mathbb{Z} \rightarrow T_{\pi}$. Then we define $\operatorname{Arg}_{\pi}:\left(\mathbb{C}^{\times}\right)^{2 n} \rightarrow(\mathbb{R} / \pi \mathbb{Z})^{2 n}=T_{\pi}^{2 n}$, and

$$
\operatorname{Arg}_{\pi}\left(z_{1}, \ldots, z_{2 n}\right)=\left(\arg _{\pi}\left(z_{1}\right), \ldots, \arg _{\pi}\left(z_{2 n}\right)\right) .
$$

We call $\mathcal{B}_{\pi}=\operatorname{Arg}_{\pi}(V) \subset T_{\pi}^{2 n}$ the rolled coamoeba of $V$.

We consider two antiholomorphic involutions on $\left(\mathbb{C}^{\times}\right)^{2 n}$ : conj, conj $j^{\prime}$ : $\left(\mathbb{C}^{\times}\right)^{2 n} \rightarrow\left(\mathbb{C}^{\times}\right)^{2 n}$ defined by

$$
\operatorname{conj}\left(z_{1}, \ldots, z_{2 n}\right)=\left(\bar{z}_{1}, \ldots, \bar{z}_{2 n}\right), \operatorname{conj}^{\prime}\left(z_{1}, \ldots, z_{2 n}\right)=\left(\frac{1}{\bar{z}_{1}}, \ldots, \frac{1}{\bar{z}_{2 n}}\right) .
$$

To each $n$-dimensional variety $V \subset\left(\mathbb{C}^{\times}\right)^{2 n}$ we associate two integer numbers. Note that if $A, B \subset\left(\mathbb{C}^{\times}\right)^{2 n}$ are two complex subvarieties of complimentary dimensions then for an open dense subset of $\epsilon \in\left(\mathbb{C}^{\times}\right)^{2 n}$ all intersection points from $A \cap \epsilon B$ are transverse and their number does not depend on $\epsilon$ (as long as it is generic). Here $\epsilon B$ stands for the coordinatewise multiplication of $B$ by $\epsilon$ in $\left(\mathbb{C}^{\times}\right)^{2 n}$ (in other words for the multiplicative translation). We define the toric intersection number $A . B \in \mathbb{Z}_{\geq 0}$ to be the number of points in $\#(A \cap \epsilon B)$ for a generic $\epsilon$ (times the corresponding multiplicities in the case when the corresponding components of $A$ or $B$ are not simple. i.e. if $A$ or $B$ are not reduced). Clearly, $A . B=B \cdot A$.

Definition 1.3. We define the conj-degree

$$
\alpha(V)=V \cdot \operatorname{conj}(V) \in \mathbb{Z}_{\geq 0}
$$

and the conj'-degree

$$
\beta(V)=V \cdot \operatorname{conj}^{\prime}(V) \in \mathbb{Z}_{\geq 0} .
$$

Definition 1.4. Let $A$ and $B$ be two smooth (differentiable) manifolds of the same dimension and $f: A \rightarrow B$ be a smooth map. We say that $f$ covers its image at most $m$ times if for any point $p \in B$ which is regular for $f$ the inverse image $f^{-1}(p)$ consists of at most $m$ points.

More generally, if $A$ is a (not necessarily smooth) real or complex algebraic variety (such as $V \subset\left(\mathbb{C}^{\times}\right)^{2 n}$ in the case when it is singular), it admits a stratification into smooth manifolds. Consider a map $f: A \rightarrow$ $B$ whose restriction $\left.f\right|_{\Sigma}$ to every stratum $\Sigma \subset A$ is smooth. Similarly, we say that $f$ covers its image at most $m$ times if for any point $p \in B$ the inverse image $f^{-1}(p)$ consists of at most $m$ points unless $p$ is a critical point for $\left.f\right|_{\Sigma}$, where $\Sigma \subset A$ is a stratum of our stratification.

1.2. Statement of the results. The main results of this paper are contained in the following theorem. 
Theorem 1. Let $V \subset\left(\mathbb{C}^{\times}\right)^{2 n}$ be an algebraic $n$-dimensional variety. Then the amoeba $\mathcal{A}(V)$ is covered by the map $\left.\log \right|_{V}$ at most $\beta(V)$ times, while the rolled coamoeba $\mathcal{B}_{\pi}(V)$ is covered by the map $\left.\operatorname{Arg}_{\pi}\right|_{V}$ at most $\alpha(V)$ times. Furthermore,

$$
\operatorname{Vol}(\mathcal{A}) \leq \frac{\pi^{2 n}}{2} \alpha(V)
$$

Note that the conventional (i.e. non-rolled) coamoeba $\mathcal{B}(V)$ cannot be covered more than the rolled coamoeba. Thus it is also covered by the coamoeba map at most $\alpha(V)$ times.

If $V$ is a complete intersection then we can easily compute the conjdegree $\alpha$ as well as the conj'-degree $\beta$ by means of the BernsteinKouchnirenko calculus as follows.

Definition 1.5. We say that

$$
V=\bigcap_{j=1}^{n} V_{j} \subset\left(\mathbb{C}^{\times}\right)^{2 n}
$$

is a toric complete intersection of hypersurfaces $V_{1}, \ldots, V_{n} \in\left(\mathbb{C}^{\times}\right)^{2 n}$ if

$$
V=\lim _{\epsilon_{j} \rightarrow 0} \bigcap_{j=1}^{n} \epsilon_{j} V_{j} .
$$

Here the limit is taken in the sense of Hausdorff metric on the subsets of $\left(\mathbb{C}^{\times}\right)^{2 n}$ (with a group invariant metric) and $\epsilon_{j}$. In particular, we require this limit to exist.

Proposition 2. Suppose that $V=\bigcap_{j=1}^{n} V_{j} \subset\left(\mathbb{C}^{\times}\right)^{2 n}$ is a complete intersection of hypersurfaces $V_{j}$ with Newton polyhedra $\Delta_{j} \subset \mathbb{R}^{2 n}$, $j=1, \ldots, n$. Then we have

$$
\alpha(V)=\operatorname{Vol}\left(\Delta_{1}, \ldots, \Delta_{n}, \Delta_{1}, \ldots, \Delta_{n}\right)
$$

and

$$
\beta(V)=\operatorname{Vol}\left(-\Delta_{1}, \ldots,-\Delta_{n}, \Delta_{1}, \ldots, \Delta_{n}\right) .
$$

Here Vol stands for the mixed volume of $2 n$ polyhedra in $\mathbb{R}^{2 n}$.

Remark 1.6. Proposition 2 and Theorem 1 produce upper bounds for the volumes of amoebas in the case of toric complete intersections in terms of the mixed volumes of the corresponding Newton polyhedra. Such bounds were conjectured in the talk by Mounir Nisse on the memorial conference for Mikael Passare in Summer 2013. Finiteness of $\operatorname{Vol}(\mathcal{A})$ was observed in [9]. 
Proof. Note that $\operatorname{conj}(V)$ is a also a toric complete intersection defined by the polynomials with the same Newton polyhedra, but conjugate coefficients while $\operatorname{conj}^{\prime}(V)$ is a toric complete intersection defined by the polynomials with $-\Delta_{j}$ as their Newton polyhedra as we need to make a substitution $z_{j} \mapsto \frac{1}{z_{j}}$ before conjugation. The proposition now follows from the Bezout theorem in the form of Bernstein-Kouchnirenko [1], 6].

In particular, if $V$ is a toric complete intersection with

$$
\Delta_{1}=\cdots=\Delta_{n}=\left\{\left(x_{1}, \ldots, x_{2 n}\right) \in \mathbb{R}_{\geq 0}^{2 n} \mid \sum_{j=1}^{2 n} x_{j} \leq 1\right\}
$$

then $\alpha(V)=1$ and $\beta(V)=\frac{(2 n) !}{(n !)^{2}}$ so Theorem 1 has the following corollary.

Corollary 3. If $V=\bar{V} \cap\left(\mathbb{C}^{\times}\right)^{2 n}$, and $\bar{V}$ is a complete intersection of hypersurfaces of degrees $d_{1}, \ldots, d_{n}$ in $\mathbb{C P}^{2 n}$ then $\mathcal{A}(V)$ is covered by the amoeba map at most $\frac{(2 n) !}{(n !)^{2}} \prod_{j=1}^{n} d_{j}^{2}$ while $\mathcal{B}_{\pi}(V)$ (as well as $\mathcal{B}(V)$ itself) is covered at most $\prod_{j=1}^{n} d_{j}^{2}$ times by the coamoeba map. Furthermore,

$$
\operatorname{Vol}(\mathcal{A}) \leq \frac{\pi^{2 n}}{2} \prod_{j=1}^{n} d_{j}^{2}
$$

\section{Proof of THE THEOREM}

\subsection{Bounds for the number of inverse images for the amoeba} and coamoeba maps. In this section we prove the first part of Theorem 1 establishing bounds for the number of inverse images of Log $\left.\right|_{V}$ and $\left.\operatorname{Arg}_{\pi}\right|_{V}$.

Note that $\left(\left.\operatorname{Arg}_{\pi}\right|_{V}\right)^{-1}(0)=V \cap\left(\mathbb{R}^{\times}\right)^{2 n} \subset\left(\mathbb{C}^{\times}\right)^{2 n}$. We may compare this with $\left(\left.\operatorname{Arg}\right|_{V}\right)^{-1}(0)=V \cap\left(\mathbb{R}_{>0}\right)^{2 n}$ in the case of the conventional (not rolled) coamoeba map. Similarly, for $p \in T_{\pi}^{2 n}=(\mathbb{R} / \pi \mathbb{Z})^{2 n}$ we have

$$
\left(\left.\operatorname{Arg}_{\pi}\right|_{V}\right)^{-1}(p)=V \cap e^{i p}\left(\mathbb{R}^{\times}\right)^{2 n} \subset\left(\mathbb{C}^{\times}\right)^{2 n}
$$

where $e^{i p} \in\left(\mathbb{C}^{\times}\right)^{2 n}$ is obtained by coordinatewise exponentiating of $i p$. If $p$ is a regular value of $\left.\operatorname{Arg}_{\pi}\right|_{V}$ then $V$ intersects $e^{i p}\left(\mathbb{R}^{\times}\right)^{2 n}$ transversally. This means that every stratum in a stratification of $V$ into smooth manifolds intersects $e^{i p}\left(\mathbb{R}^{\times}\right)^{2 n}$ transversally. By the dimension considerations, the top-dimensional stratum intersects $e^{i p}\left(\mathbb{R}^{\times}\right)^{2 n}$ in finitely many points while smaller-dimensional strata are disjoint from $e^{i p}\left(\mathbb{R}^{\times}\right)^{2 n}$. 
Furthermore, we have the inclusion

$$
\left(\left.\operatorname{Arg}_{\pi}\right|_{V}\right)^{-1}(p)=V \cap e^{i p}\left(\mathbb{R}^{\times}\right)^{2 n} \subset V \cap e^{2 i p} \operatorname{conj}(V)
$$

as $e^{i p}\left(\mathbb{R}^{\times}\right)^{2 n} \subset\left(\mathbb{C}^{\times}\right)^{2 n}$ is the invariant locus for the complex conjugation

$$
\left(z_{1}, \ldots, z_{2 n}\right) \mapsto e^{2 i p}\left(z_{1}, \ldots, z_{2 n}\right)
$$

in $\left(\mathbb{C}^{\times}\right)^{2 n}$. Thus the cardinality of $\left(\left.\operatorname{Arg}_{\pi}\right|_{V}\right)^{-1}(p)$ for regular $p$ is bounded by $\alpha(V)$ as stated in Theorem 1 .

Similarly, for a regular value $q \in \mathbb{R}^{2 n}$ of $\left.\log \right|_{V}$ we have a finite number of points in $\left(\left.\log \right|_{V}\right)^{-1}(q)$ as well as the inclusion

$$
\left(\left.\log \right|_{V}\right)^{-1}(q)=V \cap e^{q} S \subset V \cap e^{2 q} \operatorname{conj}^{\prime}(V),
$$

where $S \subset\left(\mathbb{C}^{\times}\right)^{2 n}$ is the unit torus (the fixed point locus of conj'). Thus the cardinality of $\left(\left.\log \right|_{V}\right)^{-1}(q)$ for regular $p$ is bounded by $\beta(V)$ as stated in Theorem 1.

2.2. Estimating the volume of amoeba. To finish the proof of Theorem 1 we consider the real-valued $2 n$-form on $\left(\mathbb{C}^{\times}\right)^{2 n}$

$$
\omega=\prod_{j=1}^{2 n} d x_{j}-\prod_{j=1}^{2 n} d y_{j}
$$

Here product stands for the exterior product of differential forms.

Lemma 4. We have $\left.\omega\right|_{V} \equiv 0$.

Proof. We may write

$$
\omega=\frac{1}{2^{2 n}}\left(\prod_{j=1}^{2 n}\left(d z_{j}+d \bar{z}_{j}\right)-(-1)^{n} \prod_{j=1}^{2 n}\left(d z_{j}-d \bar{z}_{j}\right)\right) .
$$

The right-hand side of this expression is the sum of monomials of degree $2 n$ in $d z_{j}$ and $d \bar{z}_{k}$. Note that if $n$ is odd then there are no monomials with odd number of $d \bar{z}_{j}$. Similarly, if $n$ is even then there are no monomials with even number of $d \bar{z}_{j}$. Thus the right-hand side of (2.4) contains only monomials where either the number of $d z_{j}$ is more than $n$ or the number of $d \bar{z}_{k}$ is more than $n$. Thus, $\omega$ must vanish everywhere on a holomorphic $n$-variety $V$.

We may consider the cardinality $\#\left(\left.\log \right|_{V}\right)^{-1}$ of the inverse image of the amoeba map as a measurable function on $\mathbb{R}^{2 n}$ (since the critical locus of $\left.\log \right|_{V}$ is nowhere dense). Then

$$
\operatorname{MultiVol}(\mathcal{A})=\int_{\mathbb{R}^{2 n}} \#\left(\left(\left.\log \right|_{V}\right)^{-1}\left(x_{1}, \ldots, x_{2 n}\right)\right) d x_{1} \ldots d x_{2 n}
$$


can be thought of as the volume of $\mathcal{A}$ taken with the multiplicities corresponding to the covering by the amoeba map. Similarly,

$$
\operatorname{MultiVol}\left(\mathcal{B}_{\pi}\right)=\int_{T_{\pi}^{2 n}} \#\left(\left(\left.A r g_{\pi}\right|_{V}\right)^{-1}\left(y_{1}, \ldots, y_{2 n}\right)\right) d y_{1} \ldots d y_{2 n}
$$

can be thought of as the volume of $\mathcal{B}_{\pi}$ taken with the multiplicities corresponding to the covering by the coamoeba map.

Corollary 5. $\operatorname{MultiVol}(\mathcal{A})=\operatorname{MultiVol}\left(\mathcal{B}_{\pi}\right)$.

Proof. Let $V_{+} \subset V$ be the open subset of $V$ where the real $2 n$-form $d x_{1} \wedge \cdots \wedge d x_{2 n}$ is non-degenerate and defines the orientation that agrees with the complex orientation of $V$. Let $V_{-} \subset V$ be the open set where these orientations disagree. Note that by Lemma 4 the form $d y_{1} \wedge \cdots \wedge d y_{2 n}$ also agrees with the complex orientation on $V_{+}$and disagrees on $V_{-}$. We have

$$
\operatorname{MultiVol}(\mathcal{A})=\int_{V_{+}} d x_{1} \wedge \cdots \wedge d x_{2 n}-\int_{V_{-}} d x_{1} \wedge \cdots \wedge d x_{2 n}
$$

while

$$
\operatorname{MultiVol}\left(\mathcal{B}_{\pi}\right)=\int_{V_{+}} d y_{1} \wedge \cdots \wedge d y_{2 n}-\int_{V_{-}} d y_{1} \wedge \cdots \wedge d y_{2 n} .
$$

The two multivolumes are equal by Lemma 4 ,

\section{Lemma 6.}

$$
\operatorname{MultiVol}(\mathcal{A})=\operatorname{MultiVol}\left(\mathcal{B}_{\pi}\right) \leq \alpha(V) \pi^{2 n}
$$

Proof. By (2.1) the cardinality of $\left(\left.\operatorname{Arg}_{\pi}\right|_{V}\right)^{-1}(p)$ is not greater than $\alpha(V)$ almost everywhere on $T_{\pi}^{2 n}$ while $\operatorname{Vol}\left(T_{\pi}^{2 n}\right)=\pi^{2 n}$.

Note that $\log :\left(\mathbb{C}^{\times}\right)^{2 n} \rightarrow \mathbb{R}^{2 n}$ is a proper map (inverse images of compact sets are compact) and thus $\left.\log \right|_{V}: V \rightarrow \mathbb{R}^{2 n}$ is also proper. Since $V$ and $\mathbb{R}^{2 n}$ are oriented manifolds of the same dimension the map $\left.\log \right|_{V}$ has a well-defined degree. Recall that this degree is equal to the number of inverse images of a generic point $q \in \mathbb{R}^{2 n}$ taken with the sign \pm 1 depending whether $\left.\log \right|_{V}$ locally preserves the orientation.

Corollary 7. The degree of the amoeba map is zero. We have

$$
\operatorname{MultiVol}(\mathcal{A})=2 \int_{V_{+}} d x_{1} \wedge \cdots \wedge d x_{2 n}=-2 \int_{V_{-}} d x_{1} \wedge \cdots \wedge d x_{2 n} .
$$

Furthermore, $\operatorname{Vol}(\mathcal{A}) \leq \frac{1}{2} \operatorname{MultiVol}(\mathcal{A})$. 
Proof. As the multivolume of $V$ is bounded by Lemma 6 we have $\mathbb{R}^{2 n} \backslash$ $\mathcal{A} \neq \emptyset$ and thus the degree of $\left.\log \right|_{V}$ must be zero. Each generic point $q \in \mathcal{A}$ is covered by $V_{+}$and $V_{-}$the same number of times.

Remark 2.1. Note that Corollary 7 immediately implies that $\beta(V)$ is always even as it coincides with the degree of the amoeba map Log $\left.\right|_{V}$ (this fact is also easy to deduce from symmetry reasons). However, as the maps $\left.\operatorname{Arg}\right|_{V}$ and $\left.\operatorname{Arg}_{\pi}\right|_{V}$ are not proper, we cannot apply the same reasoning. Note, in particular, that the parity of $\left(\left.\operatorname{Arg}\right|_{V}\right)^{-1}(p)$ is different for different generic points of $(\mathbb{R} / 2 \pi \mathbb{Z})^{2 n}$ already in the case when $V \subset \mathbb{C P}^{2}$ is a generic line (cf. e.g. [11]).

In the same time, (2.1) implies that for the parity of $\left(\left.\operatorname{Arg}_{\pi}\right|_{V}\right)^{-1}(p)$ coincides with $\alpha(V)$ for generic points $p \in T_{\pi}^{2 n}$ as non-real intersection points of $e^{-i p} V$ and $e^{i p} \operatorname{conj}(V)$ come in pairs. Thus the rolled coamoeba map has a well-defined degree mod 2 determined by $\alpha(V)$.

Corollary 7 implies that

$$
\operatorname{Vol}(\mathcal{A})=\operatorname{Vol}\left(\log \left(V_{+}\right)\right) \leq \frac{1}{2} \operatorname{MultiVol}\left(\mathcal{B}_{\pi}\right) \leq \frac{\pi^{2 n}}{2} \alpha(V) .
$$

This finishes the proof of Theorem 1 .

\section{Some REMARKS AND OPEN PROBLEMS}

3.1. Example: linear spaces in $\mathbb{C P}^{2 n}$. Let $L \subset \mathbb{C P}^{2 n}$ be an $n$ dimensional linear subspace that is generic with respect to the coordinate hyperplanes of $\mathbb{C P}^{2 n}$. Then $V=L \cap\left(\mathbb{C}^{\times}\right)^{2 n}$ can be presented as a complete intersection of hyperplanes with the Newton polyhedra given by (1.2). By Proposition 2 we have $\alpha(V)=1$. By Remark 2.1 the set $\left(\left.\operatorname{Arg}_{\pi}\right|_{V}\right)^{-1}(p)$ must consist of a single point for almost all values $p \in T_{\pi}^{2 n}$, so the inequality of Lemma 6 turns into equality. We get the following proposition.

Proposition 8 (cf. [5], [14]). If $V=\bigcap_{j=1}^{n} \subset\left(\mathbb{C}^{\times}\right)^{2 n}$ is a transverse intersection of $n$ hyperplanes

$$
H_{j}=\left\{\left(z_{1}, \ldots, z_{2 n}\right) \mid a_{j 0}+\sum_{k=1}^{2 n} a_{j k} z_{k}=0\right\}
$$

with $\prod_{j=1}^{n} \prod_{k=0}^{2 n} a_{j k} \neq 0$ then

$$
\operatorname{MultiVol}(\mathcal{A})=\operatorname{MultiVol}\left(\mathcal{B}_{\pi}\right)=\operatorname{Vol}\left(\mathcal{B}_{\pi}\right)=\pi^{2 n}
$$


In the case of $n=1$ we have $\beta(V)=2$. By Corollary 7 we have

$$
\operatorname{Vol}(\mathcal{A})=\frac{1}{2} \operatorname{MultiVol}(\mathcal{A})=\frac{\pi^{2}}{2}
$$

in this case as in [17]. This equality was used by Passare [16] to give a new proof of Euler's formula $\zeta(2)=\frac{\pi^{2}}{6}$. In the case $n>1$ we have $\beta(V)>2$, so Proposition 8 only implies the inequalities

$$
\frac{\pi^{2 n}(n !)^{2}}{(2 n !)^{2}} \leq \operatorname{Vol}(\mathcal{A}) \leq \frac{\pi^{2 n}}{2}
$$

and $\operatorname{Vol}(\mathcal{A})$ might vary with $V$. Note that our linear subspace $V \subset$ $\left(\mathbb{C}^{\times}\right)^{2 n}$ varies in a $\left(n^{2}-n\right)$-dimensional family if we identify subspaces that can be obtained from each other by multiplication by $\epsilon \in\left(\mathbb{C}^{\times}\right)^{2 n}$ (such multiplication corresponds to a translation of amoeba and thus does not change its shape or its volume).

Problem 3.1. What are the maximal and minimal possible values of $\operatorname{Vol}(\mathcal{A})$ ? It would be interesting to solve this problem already for $n=2$.

\subsection{MultiHarnack varieties in $\mathbb{R P}^{2 n}$.}

Definition 3.2. We say that an $n$-dimensional variety $V \subset\left(\mathbb{C}^{\times}\right)^{2 n}$ is multiHarnack if

$$
\operatorname{MultiVol}(\mathcal{A})=\pi^{2 n} \alpha(V)
$$

Let us recall the notion of simple Harnack curves in $\left(\mathbb{C}^{\times}\right)^{2}$ (introduced in [10]). According to the maximal volume characterization given in [13] a curve $V \subset\left(\mathbb{C}^{\times}\right)^{2}$ can be presented as $V=\epsilon C$ for a simple Harnack curve $C \subset\left(\mathbb{C}^{\times}\right)^{2}$ and a multiplicative vector $\epsilon \in\left(\mathbb{C}^{\times}\right)^{2}$ if and only if we have $\operatorname{Vol}(\mathcal{A})=\frac{\pi^{2}}{2} \alpha(V)$.

This class of curves was generalized to a larger class of curves in $\left(\mathbb{C}^{\times}\right)^{2}$, (also called multiHarnack curves) by Lionel Lang ([8], [7]). Definition 3.2 gives the multiHarnack curves in the case $n=1$.

According to Proposition 8 all generic linear spaces in $\mathbb{C P}^{2 n}$ are multiHarnack.

Problem 3.3. Do there exist multiHarnack varieties of higher degree?

Note that once $n>1$ being multiHarnack no longer implies being real even after multiplication by $\epsilon \in\left(\mathbb{C}^{\times}\right)^{2 n}$ already for linear spaces.

Remark 3.4. It might be instructive to compare Definition 3.2 against another attempt to generalize the definition of simple Harnack curves from [10] to higher dimensions. The survey [11] gave a definition of torically maximal hypersurfaces of dimension $n$ generalizing the Definition from [10] for $n=1$. However, it was recently shown (see [2]) that all torically maximal hypersurfaces in $\mathbb{R P}^{n+1}$ for $n>1$ have degree 1 . 
3.3. Foliation of $\partial \mathcal{A}$. In this subsection we suppose for simplicity that $V \subset\left(\mathbb{C}^{\times}\right)^{2 n}$ is smooth (otherwise we may restrict ourselves to the smooth part of $V$ ). Let us look at the critical locus $C \subset V$ of the map $\left.\log \right|_{V}$ and its image $D=\log (C) \subset \mathcal{A} \subset \mathbb{R}^{2 n}$ (also called the discriminant locus). We have the following generalization of Lemma 3 from [10].

Proposition 9. The set $C$ consists of the points $z \in V$ where $V$ and $z\left(\mathbb{R}^{\times}\right)^{2 n}$ are tangent.

As usual, $z\left(\mathbb{R}^{\times}\right)^{2 n}$ stands for the coordinatewise multiplication of $\left(\mathbb{R}^{\times}\right)^{2 n}$ by $z \in\left(\mathbb{C}^{\times}\right)^{2 n}$.

Proof. We have $z \in C$ iff there are vectors in $T_{z} V$ tangent to the argument torus $\log ^{-1}(q)$, where $q=\log (z)$. Any such vector multiplied by $i$ gives a vector tangent both to $V$ and $z\left(\mathbb{R}^{\times}\right)^{2 n}$, and vice versa.

Definition 3.5. Let $z \in C$. Denote

$$
F(z)=T_{z}(V) \cap T_{z}\left(z\left(\mathbb{R}^{\times}\right)^{2 n}\right) \subset T_{z}\left(\left(\mathbb{C}^{\times}\right)^{2 n}\right) .
$$

It is a real vector subspace of the tangent space $T_{z}\left(\left(\mathbb{C}^{\times}\right)^{2 n}\right)$. The rank of $z \in C$ is $\operatorname{dim}_{\mathbb{R}} F(p)$.

We denote with $C_{r} \subset C$ the locus of critical points of rank at least $r$. The following proposition follows immediately from the injectivity of $d \log$ on the tangent space to $z\left(\mathbb{R}^{\times}\right)^{2 n}$.

Proposition 10. The subspace

$$
(d \log )(F(z)) \subset T_{q}\left(\mathbb{R}^{2 n}\right)
$$

has dimension $r$ for $z \in C_{r}, q=\log (z)$.

Thus we get a preferred $r$-dimensional subspace in the tangent space of $\log (z)$ for each $z \in C_{r}$.

Let us choose a stratification of the discriminant locus $D$ to $k$ dimensional (non-closed) subvarieties $\Sigma_{k}$,

$$
D=\bigcup_{k=0}^{2 n-1} \Sigma_{k} .
$$

By a $k$-dimensional multidistribution on an open set $U$ of a manifold we mean specifying a finite set of $k$-dimensional subspaces of $T_{q} U$ for every $q \in U$ so that they depend on $q$ smoothly.

Lemma 11. For a generic point $q$ of $\Sigma_{2 n-k}$ the set $\log ^{-1}(q) \cap C_{k}$ is finite and disjoint from $C_{k+1}$. Furthermore, for each point $z \in$ $\log ^{-1}(q) \cap C_{k}$ the $k$-dimensional space $(d \log )(F(z))$ is tangent to 
$\Sigma_{2 n-k}$. We have a $k$-dimensional multidistribution (perhaps empty) of an open dense subset of $\Sigma_{2 n-k}$.

Proof. Since $\operatorname{dim} \Sigma_{k}=k$ and the rank of $d\left(\left.\log \right|_{C_{k+1}}\right)$ is at most $2 n-k-$ 1 , the image $\log \left(C_{k+1}\right)$ is nowhere dense in $\Sigma_{k}$. Also the critical values of $\left.\log \right|_{C_{k} \cap \log ^{-1}\left(\Sigma_{k}\right)}$ (treated as a map from any of its smooth stratum to the open manifold $\left.\Sigma_{2 n-k}\right)$ are nowhere dense in $\Sigma_{k}$. If $(d \log )(F(z))$ is not tangent to $\Sigma_{k} \ni \log (F(z))$ at a regular point of $\left.\log \right|_{C_{k}}$ then the rank of $d\left(\left.\log \right|_{V}\right)$ is at least $2 n-k+1$ which contradicts to the definition of $C_{k}$.

Suppose that $\mathcal{A} \subset \mathbb{R}^{2 n}$ is non-degenerate, i.e. the interior of $\mathcal{A}$ is non-empty. (This condition is equivalent to the condition $C \neq V$, i.e. to the condition that $\left.\log \right|_{V}$ has a regular point.) Then the amoeba boundary $\partial \mathcal{A}$ is a $(2 n-1)$-dimensional subset of $D$. Let us denote with $\partial_{1} \mathcal{A}$ the subset of $\partial \mathcal{A}$ formed by points $q$ such that $\log ^{-1}(q) \cap C$ consists of a single point.

Corollary 12. We have a 1-dimensional non-empty multifoliation on an open dense set in $\partial \mathcal{A}$. This is a genuine 1-dimensional foliation on an open dense set in $\partial_{1} \mathcal{A}$.

Remark 3.6. Since $V$ is $n$-dimensional (over $\mathbb{C}$ ) and $z\left(\mathbb{R}^{\times}\right)^{2 n}$ is $2 n$ dimensional (over $\mathbb{R}$ ) and totally real, the maximal dimension of $F(z)$ is $n$.

Suppose that $V=\operatorname{conj} V$, i.e. $V$ is defined over $\mathbb{R}$. Then we have $\mathbb{R} V=V \cap\left(\mathbb{R}^{\times}\right)^{2 n} \subset C_{n}$.

3.4. Dimensions greater that half. Suppose that $V$ is a $k$-dimensional algebraic variety in $\left(\mathbb{C}^{\times}\right)^{n}$ with $k>\frac{n}{2}$. Then the generic fibers of $\left.\log \right|_{V}$ and $\left.\operatorname{Arg}_{\pi}\right|_{V}$ are $n-2 k$-dimensional varieties as $V$ is $2 k$-dimensional (over $\mathbb{R}$ ) and $\mathbb{R}^{n}$ is $n$-dimensional. We may still present generic fibers of $\left.\operatorname{Arg}_{\pi}\right|_{V}$ and $\left.\log \right|_{V}$ as real algebraic varieties in a way similar to the half-dimensional case (where those fibers were points). For $p \in T_{\pi}^{n}$ and $q \in \mathbb{R}^{n}$ we consider the antiholomorphic involutions $\operatorname{conj}_{p}$, conj ${ }_{q}^{\prime}$ : $\left(\mathbb{C}^{\times}\right)^{n} \rightarrow\left(\mathbb{C}^{\times}\right)^{n}$ defined by

$$
\operatorname{conj}_{p}(z)=e^{i p}\left(\operatorname{conj}\left(e^{-i p} z\right)\right), \operatorname{conj}_{q}^{\prime}(z)=e^{q}\left(\operatorname{conj}^{\prime}\left(e^{-q} z\right)\right),
$$

where conj and conj' are defined as in (1.1):

$$
\operatorname{conj}\left(z_{1}, \ldots, z_{n}\right)=\left(\bar{z}_{1}, \ldots, \bar{z}_{n}\right), \operatorname{conj}^{\prime}\left(z_{1}, \ldots, z_{n}\right)=\left(\frac{1}{\bar{z}_{1}}, \ldots, \frac{1}{\bar{z}_{n}}\right) .
$$

Note that the fixed point set of $\operatorname{conj}_{p}$ is $\operatorname{Arg}_{\pi}^{-1}(p)=e^{i p}\left(\mathbb{R}^{\times}\right)^{n}$ while the fixed point set of $\operatorname{conj}_{q}^{\prime}$ is $\log ^{-1}(q)$. The following proposition is straightforward. 
Proposition 13. The antiholomorphic involutions $\operatorname{conj}_{p}$, conj $\mathrm{j}_{q}$ act on algebraic varieties $V \cap \operatorname{conj}_{p}(V)$ and $V \cap \operatorname{conj}_{q}^{\prime}(V)$ so that the fixed point sets are $\left(\left.\operatorname{Arg}_{\pi}\right|_{V}\right)^{-1}(p)$ and $\left(\left.\log \right|_{V}\right)^{-1}(q)$.

Thus we may think of the fibers of $\left.\operatorname{Arg}_{\pi}\right|_{V}$ and $\left.\log \right|_{V}$ as real algebraic varieties whose complexification is $V \cap \operatorname{conj}_{p}(V)$ and $V \cap \operatorname{conj}_{q}^{\prime}(V)$. For regular fibers these varieties are non-singular $(n-2 k)$-dimensional varieties near their real points.

Example 3.7. Consider the plane

$$
V=\left\{(x, y, z) \in\left(\mathbb{C}^{\times}\right)^{3} \mid 1+x+y+z=0\right\}
$$

in $\left(\mathbb{C}^{\times}\right)^{3}$. Both $V$ and $\operatorname{conj}_{p}(V)$ are planes, so fibers of $\left.\operatorname{Arg}_{\pi}\right|_{V}$ are intersections of two real planes in $\left(\mathbb{R}^{\times}\right)^{3}$ after a multiplicative translation by $e^{i p}$. For generic fibers these two planes are transversal, so their intersection is a line. For special fibers these planes might be parallel planes, or two copies of the same plane. These special cases correspond to empty or two-dimensional fibers of $\left.\operatorname{Arg} \pi\right|_{V}$.

The surface $\operatorname{conj}_{q}^{\prime}(V)$ is the image of a plane under the Cremona transformation $x \mapsto \frac{1}{x}, y \mapsto \frac{1}{y}, z \mapsto \frac{1}{z}$. For a generic $q$ the intersection of $V$ and $\operatorname{conj}_{q}^{\prime}(V)$ is a smooth elliptic curve. Its real locus may be empty, or consist of one or two circles. All three cases are realized as generic fibers of $\left.\log \right|_{V}$ for $V$ given by (3.3).

\section{REFERENCES}

[1] D. N. Bernstein. The number of roots of a system of equations. Funkcional. Anal. i Priložen., 9(3):1-4, 1975.

[2] Erwan Brugallé, Grigory Mikhalkin, and Jean-Jacques Risler. In preparation.

[3] Bo Feng, Yang-Hui He, Kristian D. Kennaway, and Cumrun Vafa. Dimer models from mirror symmetry and quivering amoebae. Adv. Theor. Math. Phys., $12(3): 489-545,2008$.

[4] I. M. Gelfand, M. M. Kapranov, and A. V. Zelevinsky. Discriminants, resultants and multidimensional determinants. Modern Birkhäuser Classics. Birkhäuser Boston Inc., Boston, MA, 2008. Reprint of the 1994 edition.

[5] Peter Johansson. Coamoebas, 2010. Licentiate Thesis, Stockholm University.

[6] A. G. Kouchnirenko. Newton polyhedra and Bezout's theorem. Funkcional. Anal. i Priložen., 10(3, 82-83.), 1976.

[7] Lionel Lang. A generalization of simple Harnack curves. arXiv:1504.07256.

[8] Lionel Lang. Geometry of tropical curves and application to real algebraic geometry. PhD Thesis, Geneva 2014.

[9] Farid Madani and Mounir Nisse. On the volume of complex amoebas. Proceedings of the AMS, 141:1113-1123, 2013.

[10] G. Mikhalkin. Real algebraic curves, the moment map and amoebas. Ann. of Math. (2), 151(1):309-326, 2000. 
[11] Grigory Mikhalkin. Amoebas of algebraic varieties and tropical geometry. In Different faces of geometry, volume 3 of Int. Math. Ser. (N. Y.), pages 257-300. Kluwer/Plenum, New York, 2004.

[12] Grigory Mikhalkin and Andrei Okounkov. Geometry of planar log-fronts. Mosc. Math. J., 7(3):507-531, 575, 2007.

[13] Grigory Mikhalkin and Hans Rullgård. Amoebas of maximal area. Internat. Math. Res. Notices, (9):441-451, 2001.

[14] Mounir Nisse and Mikael Passare. Amoebas and coamoebas of linear spaces. arXiv: 1205.2808 .

[15] Mikael Passare. Amoebas, convexity and the volume of integer polytopes. In Complex analysis in several variables-Memorial Conference of Kiyoshi Oka's Centennial Birthday, volume 42 of Adv. Stud. Pure Math., pages 263-268. Math. Soc. Japan, Tokyo, 2004.

[16] Mikael Passare. How to compute $\sum 1 / n^{2}$ by solving triangles. Amer. Math. Monthly, 115(8):745-752, 2008.

[17] Mikael Passare and Hans Rullgård. Amoebas, Monge-Ampère measures, and triangulations of the Newton polytope. Duke Math. J., 121(3):481-507, 2004.

Université de Genève, Mathématiques, Villa Battelle, 1227 Carouge, SuISSE 